Ann. Biol. anim. Bioch. Biophys., I970, 10 (4), 689-696.

\title{
COMPARAISON DE DEUX TECHNIQUES POUR DÉNOMBRER LES PROTOZOAIRES DU RUMEN ET PRÉCISION OBTENUE
}

\author{
D. SAUVANT et $\mathrm{Ph}$. GOUET * \\ Laboratoire de Recherches sur la Conservation et l'Efficacité des Aliments, \\ Centre national de Recherches zootechniques, 78-Jouy-en-Josas, \\ Institut national de la Recherche agronomique
}

\section{RÉSUMÉ}

Les protozoaires du rumen (Holotriches et Entodinomorphes) sont dénombrés au microscope en utilisant, pour des échantillons identiques, un hématimètre de Malassez ou une cellule de Petroff-Hauser. L'interprétation statistique montre que les résultats sont homogènes entre les deux types de cellule. L'emploi de l'hématimètre est plus aisé et nécessite deux fois moins de temps que la cellule de Petroff-Hauser. La méthodologie est par ailleurs précisée et une abaque permet de déterminer le nombre de cellules de Malassez à compter selon la précision que l'on désire obtenir.

On sait que les protozoaires du rumen, par leur nombre et leur taille, ont un rôle métabolique important (HUNGaTE, I966) ; toutefois, les expériences de défaunation de la panse montre que la présence des protozoaires est favorable mais non indispensable à un état nutritionnel satisfaisant du ruminant (ABOU-AKKADA, EL-SHAZLY, I965).

Au fur et à mesure que leur écologie, leurs rôles nutritionnels et leurs relations avec la population bactérienne voisine se préciseront (KURIHARA et al., I968), la quantification des différentes espèces de protozoaires deviendra de plus en plus utile pour interpréter les expériences sur la digestion dans le rumen. Le nombre des protozoaires constitue, par ailleurs, le critère de validité le plus sensible des cultures en continu de jus de rumen dont ils ont tendance à disparaittre pour une raison encore inconnue (SLYTER, I964).

* Adresse actuelle : CRZV. Theix par Saint-Genès-Champanelle, 63. 
Ces faits soulignent la nécessité de disposer d'une méthode de dénombrement de la faune de la panse qui soit simple et de précision connue.

La variabilité du gabarit des protozoaires et la turbidité du milieu ne permettent pas de les compter par néphélémétrie. Les premiers auteurs ont dénombré des protozoaires avec des hématimètres : JoHnson et al. (I944), WELLER et GRAY (1954) et Morr (I95I) avec des cellules de o,I $\mathrm{mm}$ de profondeur, et plus récemment WARNER (I956), STEWAR'T (I950) et Quinn (I962) avec des cellules de Manners de 0,2 mm de profondeur. OXFORD (I955) souligne l'inconvénient d'employer de telles cellules lorsque le gabarit des protozoaires excède $200 \mu$ en longueur et propose une technique inspirée de celle décrite par AdAm (I95I) pour compter les ciliés du cæcum du cheval. Cette méthode consiste à glisser une goutte de suspension entre une lame et une lamelle séparées de $\mathrm{I} \mathrm{mm}$ et à compter avec des champs optiques préalablement étalonnés ; elle est utilisée ensuite par Boyne et al. (I957), MoIr et al. (I957) et PurSER et al. (r959). Par ailleurs, Hungate (I942) et GutTrERrez (I955) dénombrent les protozoaires aspirés dans un tube capillaire. Plus récemment, RUFENER (I963) et SiYTER (r964) comptent les ciliés du rumen contenus dans le volume limité par 2 couvercles de cellules de Petroff-Hauser superposés.

Notre travail a pour objet d'évaluer la précision des techniques d'échantillonnage puis de comparer la technique de comptage avec hématimètre à la plus récente avec couvercle de cellule de Petroff-Hauser. I1 se propose aussi, éventuellement, de mettre en évidence des différences significatives entre des animaux, des régimes et des heures de prélèvement.

\section{MATÉRIEL E'T MÉTHODE}

Le matériel animal se compose de 6 moutons Ile-de-France porteurs de fistule du rumen recevant 2 à 2 les régimes expérimentaux suivants :

$$
\begin{aligned}
& \mathrm{R}_{1}: 500 \mathrm{~g} \text { Orge }+500 \mathrm{~g} \text { paille d'avoine }+20 \mathrm{~g} \text { Urée } \\
& \mathrm{R}_{2}: 500 \mathrm{~g} \text { Maïs }+500 \mathrm{~g} \text { paille d'avoine }+20 \mathrm{~g} \text { Urée } \\
& \mathrm{R}_{3}: 500 \mathrm{~g} \text { Manioc }+500 \mathrm{~g} \text { paille d'avoine }+30 \mathrm{~g} \text { Urée }
\end{aligned}
$$

Trois séries de I 8,6 et 6 prélèvements de contenu de rumen sont effectuées après 7 , Io et I3 semaines d'accoutumance à ces régimes. Dans la première série, constituant l'expérience principale, les prélèvements sont faits avec chaque mouton à jeun, puis 3 heures et 5 heures après le repas $\left(\mathrm{H}_{0}, \mathrm{H}_{3}, \mathrm{H}_{5}\right.$ du tabl. $\left.\mathbf{I}\right)$. A chaque fois, 200 à $300 \mathrm{ml}$ de contenu de rumen sont pompés de manière à constituer un échantillon aussi représentatif que possible et mis dans des flacons immédiatement bouchés. A partir de chaque flacon préalablement agité constituant l'échantillon primaire (tabl. $\mathrm{I}: \mathrm{A}_{1}, \mathrm{~B}_{1} \ldots$ ), 2 échantillons secondaires ( $a$ et $b$ ) de $\mathrm{I} \mathrm{ml}$ de contenu de rumen, sont rapidement prélevés avec une pipette $(\mathrm{I} \mathrm{ml}$ ) dont l'extrémité est sciée, mélangés ensuite à $3 \mathrm{ml}$ d'une solution contenant $1 / 3$ de glycérol, $2 / 3$ d'eau physiologique dans des proportions différentes suivant les deux méthodes de dénombrement employées.

L'expérience principale a consisté à comparer les deux techniques de numération à partir de 5 comptages effectués sur chaque échantillon secondaire; 4 avec l'hématimètre de Malassez et I dans un couvercle de cellule de Petroff-Hauser.

- L'hématimètre de Malassez (profondeur $0,2 \mathrm{~mm}$ ) comporte côte à côte, 2 grilles identiques, $B$ et $H$, gravées sur le verre. Chaque grille délimite une surface de $5 \mathrm{~mm}^{2}$ et permet ainsi d'évaluer le nombre de protozoaires contenus dans $\mathrm{I} \mathrm{mm}^{3}$ de suspension. Pour obtenir 4 dénombrements, chaque grille est remplie deux fois de suite ( $\alpha$ et $\beta$ ), avec la dilution I/ 6 de chaque échantillon secondaire. La pipette Pasteur, utilisée pour ces opérations, dont l'extrémité est sciée et non cassée, doit être maintenue horizontale sitôt le prélèvement effectué et avec un angle d'incidence de $65^{\circ}-70^{\circ}$ au moment de l'écoulement pour éviter une décantation rapide des protozoaires qui ris- 
querait de fausser les résultats. A chaque fois, la grille $\mathrm{B}$ reçoit le bas de la pipette et la grille $\mathrm{H}$ le haut, pour pouvoir s'assurer de la validité de la technique par comparaison statistique des résultats.

TABLEA U I

Schéma de l'expérience principale

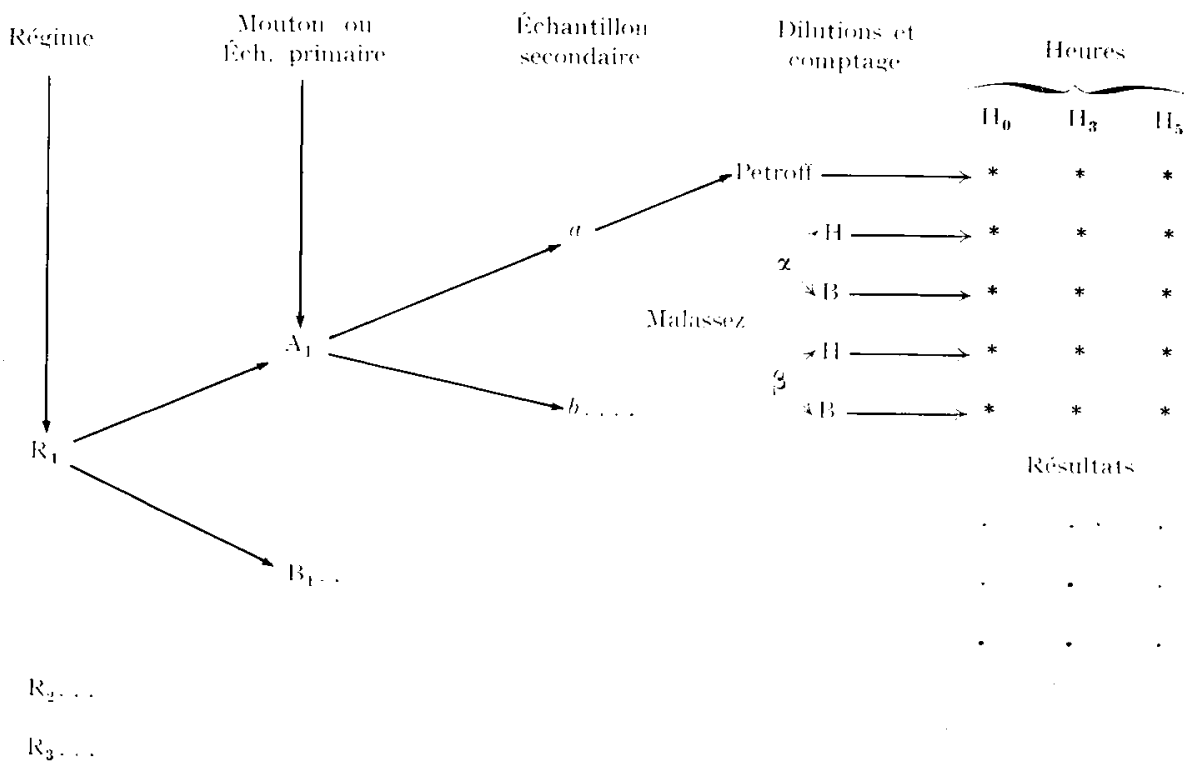

- Le couvercle de la cellule de Petroff-Hauser est rempli avec la dilution $1 / 640$ du jus de rumen et recouvert à l'aide d'un second couvercle.

Schéma:

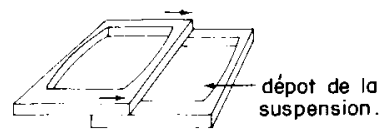

Le volume ainsi isolé est préalablement évalué à l'aide d'une seringue micrométrique et tous les protozoaires qu'il contient sont comptés après décantation.

Les examens microscopiques sont faits avec un grossissement de 60 et portent sur 2 grandes familles: Holotriches et Entodinomorphes.

L'ensemble des informations fournies par les 264 résultats est traité suivant cinq analyses successives:

- Influence de la taille des protozoaires sur leur répartition dans l'hématimètre.

- Influence de la position de la pipette lors du dépôt des échantillons.

- Comparaison des deux méthodes de dénombrement.

- Précision des dénombrements sur l'hématimètre de Malassez.

- Répétabilité des résultats obtenus d'un prélèvement à l'autre chez un même animal.

\section{RÉSULTATS}

Le nombre moyen de protozoaires est environ de I50. $\mathrm{IO}^{4} / \mathrm{ml}$. Les Holotriches n'excèdent pas $2 \%$ du nombre des Entodinomorphes : cette répartition est peutêtre due à la part importante de substances amylacées dans les glucides de la ration. 
Les proportions des sous-espèces d'Entodinomorphes varient d'un animal à l'autre mais semblent se maintenir constantes pour un même animal. Les Holotriches se répartissent environ par moitié entre les genres Isotricha et Dasytricha.

TABLEAU 2

Nombres moyens de protozoaires obtenus

$\grave{a}$ partir d'échantillons identiques $\left(1 \mathrm{I}^{2} / \mathrm{ml}\right)$

\begin{tabular}{|c|c|c|c|}
\hline & $\begin{array}{c}\text { Cellule de } \\
\text { Petroff }\end{array}$ & $\begin{array}{c}\text { Hématimètre } \\
\text { de Malassez }\end{array}$ & Total \\
\hline Holotriches & 1,59 & 2,08 & 3,67 \\
\hline Entodinomorphes ....... & 153,09 & 117.77 & 300,86 \\
\hline Total .... & 154,68 & 149,85 & 304,53 \\
\hline
\end{tabular}

\section{Validité des dénombrements sur hématimètre}

Deux facteurs sont susceptibles de fausser les résultats obtenus avec 1'hématimètre : une répartition inégale des protozoaires due aux différences de taille, une inclinaison de la pipette au moment du dépôt de l'échantillon.

\section{Influence de la taille des protozoaires sur leur répartition.}

Cette répartition n'est pas uniforme dans le couvercle de la cellule de Petroff, mais ce fait n'a pas d'influence puisque tous les protozoaires y sont comptés. Par contre, pour les numérations sur hématimètre, la goutte de suspension étant déposée au milieu de la grille, on risque de voir les Holotriches, de gabarit moyen, rester au centre au moment où la lamelle est posée et fausser ainsi la répartition entre les deux espèces.

Il est donc nécessaire de vérifier par un test $\chi^{2}$ si le rapport Holotriches/Entodinomorphes reste homogène d'une technique à l'autre.

Le résultat du test est $\chi^{2}=0,058$; la limite de signification du $\chi^{2}$ étant de 3,84 au niveau de $5 \mathrm{p}$. Ioo, la répartition entre les deux espèces pour un même échantillon est la même quelle que soit la méthode de dénombrement choisie.

\section{Infuence de la position de la pipette lors du dépôt des échantillons}

Une pipette tenue trop verticalement entraîne, d'une part, une surestimation du nombre total de protozoaires et d'autre part, un nombre moyen supérieur dans la grille $\mathbf{B}$.

Pour s'assurer de la validité de la méthode, il faut comparer les I44 résultats obtenus par moitié entre les grilles $\mathrm{B}$ et $\mathrm{H}$.

Par ailleurs, le schéma d'analyse factorielle $2 \times 2$ adopté, permet de mettre simultanément en évidence des différences éventuellement significatives entre les prélèvements $\alpha$ et $\beta$ provenant d'un même échantillon secondaire.

Les valeurs de la loi $\mathrm{F}$ obtenues pour ces 2 facteurs de variation sont très inférieures au seuil de signification au niveau 5 p. Ioo. Ces I44 résultats sont donc statis- 
tiquement homogènes et peuvent être groupés 4 par 4 pour réduire le nombre de degrés de liberté de l'analyse suivante.

\section{Comparaison des deux méthodes de dénombrement}

Elle est effectuée à partir de 72 résultats des 36 échantillons secondaires obtenus avec les dénombrements sur couvercle de cellule de Petroff-Hauser et les résultats groupés de l'hématimètre.

Le dispositif expérimental adopté permet d'étudier dans la même analyse de variance, quatre autres facteurs de dispersion : la prise des échantillons secondaires, les 6 animaux, les 3 heures de prélèvement, les 3 régimes.

TABLEAU 3

Résultats de l'analyse de variance

\begin{tabular}{c|c}
\hline Facteur de variation & Signification $\left.{ }^{1}{ }^{1}\right)$ \\
\hline Technique de dénombrement & N.S. \\
\hline Prélèvement secondaire & N.S. \\
\hline Animaux & Négimes \\
\hline Heures de prélèvement & $*$ \\
\hline
\end{tabular}

(1) N.S. : Non significatif au niveau 5 p. 100.

* : Significatif au niveau 5 p. 100.

** : Significatif au niveau 1 p. 100.

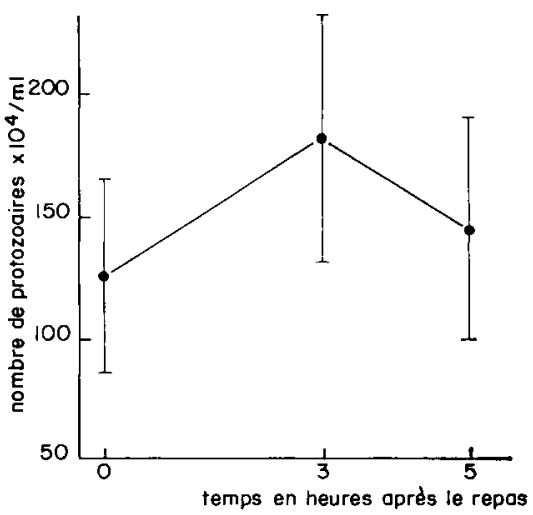

Fig. I. - Evolution postprandiale du nombre moyen de protozoaires dans le rumen de mouton

Le tableau 3 révèle 1'homogénéité des résultats des méthodes de dénombrement et d'échantillonnage et souligne l'évolution postprandiale significative du nombre moyen de protozoaires (fig. I). Par ailleurs, 1'absence de différences significatives. 
entre les régimes, provient de la part importante de la variance totale prise par les variations individuelles. (On a pu ainsi observer des variations de l'ordre de I à Io entre 2 animaux recevant le même régime.)

\section{Précision des dénombrements sur hématimètre de Malassez}

La précision des dénombrements est estimée en comptant 30 cellules soit 60 grilles $\mathrm{H}$ ou $\mathrm{B}$ à partir d'un même échantillon secondaire prélevé 5 heures après le repas. La fonction de distribution des résultats obtenus a un coefficient de variation de I4 p. Ioo ce qui permet de tracer les abaques représentant pour les niveaux 5 p. Ioo, ro p. Ioo et 20 p. Ioo la longueur de l'intervalle de confiance en p. Ioo de lą moyenne et en fonction du nombre de cellules dénombrées (fig. 2).

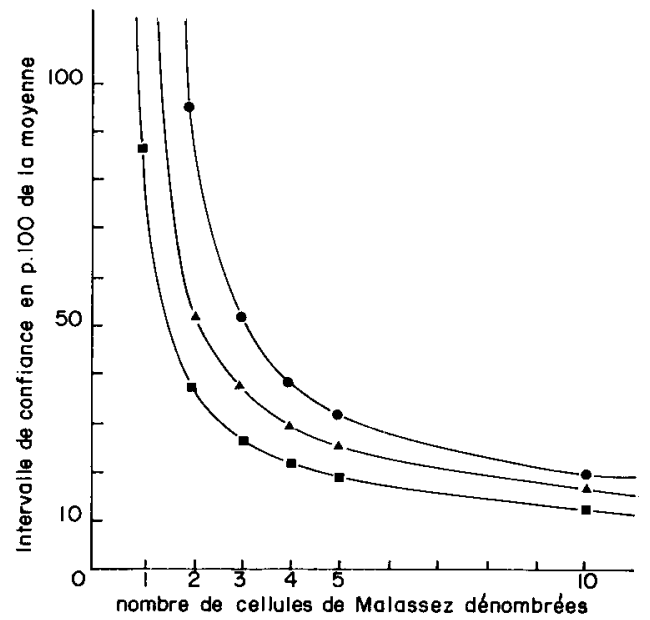

FIG. 2. - Précision d'un dénombrement de protozoaìes du rumen sur cellules de Malassez pour les niveaux 5 p. $100:-\bullet-\bullet ;$ 10 p. $100:-\Delta-\Delta$ et 20 p. $100-\square-$ -

\section{Répétabilité des résultats obtenus d'un prélèvement à l'autre chez un même animal}

I,es résultats présentés jusqu'ici sont enregistrés à partir de prélèvements effectués le même jour. Il est intéressant de connaître la répétabilité du nombre de protozoaires présents chez un animal prélevés dans les mêmes conditions à plusieurs jours d'intervalle. Dans ce but, deux autres séries de prélèvements sont effectués 3 et 6 semaines après la première, 5 heures après le repas et les corrélations respectives entre les 3 séries de 6 résultats sont calculées:

\section{Corrélation entre la $I^{\text {re }}$ et la $2^{\mathbf{e}}$ série $+0,245$ \\ Corrélation entre la $\mathrm{I}^{\mathrm{re}}$ et la $3^{\mathrm{e}}$ série : $+0,292$ \\ Corrélation entre la $2^{\mathrm{e}}$ et la $3^{\mathrm{e}}$ série : $+0,57 \mathrm{I}$}

Ces corrélations sont positives mais le nombre réduit de données fait qu'aucun de ces coefficients n'est significatif au niveau 5 p. Ioo. 


\section{DISCUSSION ET CONCLUSIONS}

Une étude préalable a mis en évidence une différence significative entre les résultats des grilles $\mathrm{B}$ et $\mathrm{H}$ de l'hématimètre. Nous avons pu observer que cet inconvénient est supprimé en tenant la pipette de prélèvement avec un angle d'incidence de $65^{\circ}-70^{\circ}$ au moment du dépôt de 1'échantillon, ce qui permet aux protozoaires de couler avec le flot sans se décanter au bas de la pipette. L'homogénéité des deux méthodes de numération vis-à-vis de l'ensemble des résultats, joue en faveur de l'hématimètre qui est d'emploi plus aisé et nécessite deux fois moins de temps pour arriver à des résultats comparables. Par ailleurs, l'absence de différences significatives entre les résultats obtenus à partir des échantillons $a, b$ et $\alpha, \beta$, signifie qu'un seul prélèvement peut suffire dans la mesure où l'échantillon précédent a été correctement homogénéisé.

Cette étude permet d'évaluer directement le nombre de cellules de Malassez à dénombrer pour obtenir une précision déterminée et révèle qu'un minimum de 4 à 5 dénombrements est nécessaire pour obtenir une méthode suffisamment sensible.

Les variations individuelles hautement significatives entre les animaux empêchent de mettre en évidence des différences entre les régimes. Ce résultat souligne le risque d'erreur que présente l'utilisation d'un seul animal comme donneur de jus de rumen pour des études métaboliques et surtout microbiologiques dans le rumen. Pour comparer l'influence des régimes sur le nombre des protozoaires (ce qui n'était pas le but), l'expérience aurait dû être menée en carré latin afin de maîtriser la part importante de la variance individuelle, et avec des prélèvements répétés sur plusieurs jours dans les mêmes conditions, pour réduire les variations des résultats enregistrés pour un même animal à différents jours.

En conclusion, la technique de dénombrement des protozoaires du rumen avec l'hématimètre de Malassez, permet d'obtenir des résultats distribués avec un coefficient de variation d'environ I4 p. Ioo, à condition de respecter les conditions suivantes :

- Homogénéiser soigneusement l'échantillon de jus de rumen.

- Prélever les échantillons aussitôt après homogénéisation des suspensions.

- Maintenir la pipette dans les positions préconisées : horizontale aussitôt après le prélèvement et avec un angle d'incidence de $65^{\circ}-70^{\circ}$ au moment du dépôt de la suspension sur les grilles de l'hématimètre.

Reçu pour publication en juillet 1970.

\section{SUMMARY}

COMPARATIVE VALUE AND ACCURACY OF TWO TECHNIQUES

FOR COUNTING RUMEN PROTOZOA

Rumen protozoa (Holotrichs and Entodinomorphs) were counted by microscopic exam1nation, either with a Petroff-Hauser counting cell or with a haemocytometer chamber (Malassez $0.02 \mathrm{~mm}$ deep).

The rumen juice diluted to a suitable volume in a formalin and glycerol-buffer medium. 
before counting. A great difficulty in counting protozoa lies in their fast sedimentation to the bottom of the suspension. The medium must be strongly fanned during transfers and sampling, and as soon as the sample has been pipetted, the pipette must be held at $65^{-70^{\circ}}$ incidence to prevent sedimentation of protozoa, allowing them to flow down into the counting chamber.

The use of the haemocytometer process is twice faster and less tedious than the PetreffHauser. The former method is described in this paper and a chart enables to estimate its accuracy depending on the number of Malassez cells counted.

\section{RÉFÉRENCES BIBLIOGRAPHIQUES}

Abou-Akkada A. R., El-Shazly K., I964. Effect of absence of Ciliate Protozoa from the rumen on microbial activity and growth of lambs. Appl. Microbiol., 12, 384-390.

Adam K. M. G., I95I. The quantity and distribution of the Ciliate Protozoa in the large intestine of the horse. Parasitology. 41, 301-309.

Boyne A. W., Eadie J. M., RaitT K., I957. The development and testing of a method of counting rumen Ciliate Protozoa. J. Gen. Microbiol., 17, 4I4-423.

GutTIERREZ J., I955. Experiments on the culture and physiology of holotrichs from the bovine rumen. Biochem. J., 60, 516-522.

Hungate R. E., I94I. The culture of Euplodinium neglectum, with experiments on the digestion of cellulose. Biol. Bull., 83, 303-319.

Hungate R. E., I966. The rumen and its microbes New York and Academic Press.

Johnson B. C., Hamilton R. S., Robinson W. R., Garky J. C., I944. On the mechanism of non protein nitrogen utilization by ruminants. J. Anim. Sci., 3, 287-298.

Kurihara Y., Eadie Margaret, J., Hobson P. N., ManN J. O., 1968. Relation between Bacteria and Ciliate Protozoa in the sheep rumen. J. Gen. Microbiol., 51, 287-298.

Morr R. J., I95I. The seasonal variation in the ruminal micro-organisms of grazing sheep. Aust. J. A gric. Res., 2, 322-330.

Moir R. J., Somers M., Ig66. A factor influencing the protozoal population in sheep. Nature, I.ond., 178, I 472 .

Oxford A. E., I955 a. The rumen ciliate Protozoa ; their chimical composition metabolism, requirements for maintenance and culture, and physiological significance for the host. Exp. Parasitol., 4, $569-605$.

Purser D. B., Moir R. J., I959. Ruminal flora studies in the sheep. IX. The effect of pH on the ciliate population of the rumen in vitro. Aust. J. A gric. Res., 10, 555-564.

Quinn L. Y., Burroughs W., Christiansen W. C., ig62. Continuous culture of ruminal microorganisms in chemically defined medium. II. Culture medium studies. Appl. Microbiol., 10, 583-592.

Rufener W. H., Nelson W. O., Wolin M. J., ig63. Maintenance of the rumen microbia population in continuous culture. Appl. Microbiol., 11, I96-20I.

Slyter L. L., Nelson W. O., Wollin M. J., I964. Modification of a device for maintenance of the rumen microbial population in continuous culture. Appl. Microbiol., 12, 374-377.

Stewart D. G., Warner A. C. I., Seeley, r96o. Continuous culture as a method for studying rumen fermentation. Appl. Microbiol., 9, $150-156$.

Warner A. C. I., 1956. Criteria for establishing the validity of in vitro studies with rumen microorganisms in so called artificial rumen systems. J. Gen. Microbiol., 14, 733-748.

IVELlFR R. A., GRAY F. V., I954. The passage of starch through the stomach of the sheep. J. Exp. Biol, , 31, 40-48. 